\title{
Allergen immunotherapy for allergic rhinoconjunctivitis: protocol for a systematic review
}

\author{
Sangeeta Dhami ${ }^{* *}$ (D) Ulugbek Nurmatov ${ }^{2}$, Graham Roberts ${ }^{3,4}$, Oliver Pfaar ${ }^{5}$, Antonella Muraro ${ }^{6}$, \\ Ignacio J. Ansotegui ${ }^{7}$, Moises Calderon ${ }^{8}$, Cemal Cingi ${ }^{9}$, Pascal Demoly ${ }^{10}$, Stephen Durham ${ }^{8}$, \\ Ronald Gerth van Wijk ${ }^{11}$, Susanne Halken ${ }^{12}$, Eckard Hamelmann ${ }^{13}$, Peter Hellings ${ }^{14}$, Lars Jacobsen ${ }^{15}$, \\ Edward Knol ${ }^{16}$, Desiree Larenas Linnemann ${ }^{17}$, Sandra Lin ${ }^{18}$, Vivian Maggina ${ }^{19}$, Hanneke Oude-Elberink $^{20}$, \\ Giovanni Pajno ${ }^{21}$, Ruby Panwankar ${ }^{22}$, Elideanna Pastorello ${ }^{23}$, Constantinos Pitsios ${ }^{24}$, Giuseppina Rotiroti ${ }^{25}$, \\ Frans Timmermans ${ }^{26}$, Olympia Tsilochristou ${ }^{27}$, Eva-Maria Varga ${ }^{28}$, Jamie Wilkinson ${ }^{29}$, Andrew Williams ${ }^{30}$, \\ Margitta Worm ${ }^{31}$, Luo Zhang ${ }^{32}$ and Aziz Sheikh ${ }^{33}$
}

\begin{abstract}
Background: The European Academy of Allergy and Clinical Immunology (EAACI) is in the process of developing the EAACI Guidelines for Allergen Immunotherapy (AIT) for the Management of Allergic Rhinoconjunctivitis. We seek to critically assess the effectiveness, cost-effectiveness and safety of AIT in the management of allergic rhinoconjunctivitis.

Methods: We will undertake a systematic review, which will involve searching international biomedical databases for published, in progress and unpublished evidence. Studies will be independently screened against pre-defined eligibility criteria and critically appraised using established instruments. Data will be descriptively and, if possible and appropriate, quantitatively synthesised.
\end{abstract}

Conclusion: The findings from this review will be used to inform the development of recommendations for EAACI's Guidelines on AIT.

Keywords: Allergy, Allergic rhinoconjunctivitis, Allergen immunotherapy, Rhinitis

\section{Background}

Allergic rhinoconjunctivitis is a very common chronic condition that can result in considerable morbidity and impairment of quality of life [1-3]. The disease is triggered by exposure to seasonal and/or perennial allergens and, depending on the nature of the allergenic trigger(s) and patterns of exposure, symptoms may be persistent or intermittent [4]. Allergic rhinitis is typically characterized by symptoms of nasal obstruction, a watery nasal discharge, sneezing and itching, and there is often (but not invariably) involvement of the conjunctiva, which

\footnotetext{
*Correspondence: sangeetadhami@hotmail.com

${ }^{1}$ Evidence-Based Health Care Ltd, Edinburgh, UK

Full list of author information is available at the end of the article
}

manifests with itching, injection and tearing [5]. There may in addition be an impact on the ability to concentrate, on school and work performance, $[6,7]$ and interference with daily activities and sleep; furthermore, allergic rhinitis is a risk factor for the development of asthma [8].

Symptoms can, in many cases, be controlled with avoidance measures and conventional therapy such as oral, intranasal and intraocular $\mathrm{H} 1$-antihistamines, intranasal corticosteroids and anti-leukotrienes, as mono-therapy or in combination $[4,9,10]$. Allergen immunotherapy (AIT) is an additional treatment option, particularly for those with more troublesome disease which remains inadequately controlled by avoidance and 
pharmacotherapy [11-13]. The problem of uncontrolled rhinitis, despite treatment, continues to represent a therapeutic challenge in some patients [14].

The European Academy of Allergy and Clinical Immunology (EAACI) is in the process of developing the EAACI Guidelines for AIT, and this systematic review is one of five inter-linked evidence syntheses that are being undertaken in order to provide a state-of-the-art synopsis of the current evidence base in relation to evaluating AIT for the treatment of allergic rhinoconjunctivitis, food allergy, venom allergy and allergic asthma, and allergy prevention, which will be used to inform the formulation of key clinical recommendations. This review will focus on the effectiveness of AIT using key patient relevant outcomes: symptom and/or medication scores and disease specific quality of life [15]. It will also examine the cost-effectiveness and safety of AIT in the management of allergic rhinoconjunctivitis.

\section{Methods}

\section{Search strategy}

A highly sensitive search strategy has been developed, and validated study design filters will be applied to retrieve articles pertaining to the use of AIT for allergic rhinoconjunctivitis from electronic bibliographic databases. We have conceptualized the search to incorporate the four elements shown in Fig. 1.

To retrieve systematic reviews, we will use the systematic review filter developed at McMaster University Health Information Research Unit (HIRU) (http://www. hiru.mcmaster.ca/hiru/HIRU_Hedges_MEDLINE_Strategies.aspx\#Reviews). To retrieve randomized controlled trials (RCTs), we will apply the Cochrane highly sensitive search strategy for identifying RCTs in MEDLINE [16]. To retrieve case series, we will use the filter developed by librarians at Clinical Evidence: http://clinicalevidence. bmj.com/x/set/static/ebm/learn/665076.html.
We will search the following databases:

- Cochrane Library including,

- Cochrane Database of Systematic Reviews (CDSR)

- Database of Reviews of Effectiveness (DARE)

- CENTRAL (Trials)

- Methods Studies

- Health Technology Assessments (HTA)

- Economic Evaluations Database (EED)

- MEDLINE (OVID)

- Embase (OVID)

- CINAHL (Ebscohost)

- ISI Web of Science (Thomson Web of Knowledge)

- TRIP Database (http://www.tripdatabase.com)

- Clinicaltrials.gov (NIH web).

- Clinical trials register (http://www.clinicaltrialsregister.eu) launched by the European Medicines Agency (EMA)

- Current controlled trials (http://www.controlled-trials.com)

- Australian and New Zealand Clinical Trials Registry (http://www.anzctr.org.au).

The search strategy has been developed on OVID MEDLINE and then adapted for the other databases (see Appendix 1). In all cases, the databases will be searched from inception to October 31, 2015. Additional references will be located through searching the references cited by the identified studies, and unpublished work, while research in progress will be identified through discussion with experts in the field. We will invite experts who are active in the field from a range of disciplines and regions to add to the list of included studies by identifying additional published and unpublished papers they are aware of and research in progress. There will be no language restrictions employed; where possible, relevant literature will be translated into English.
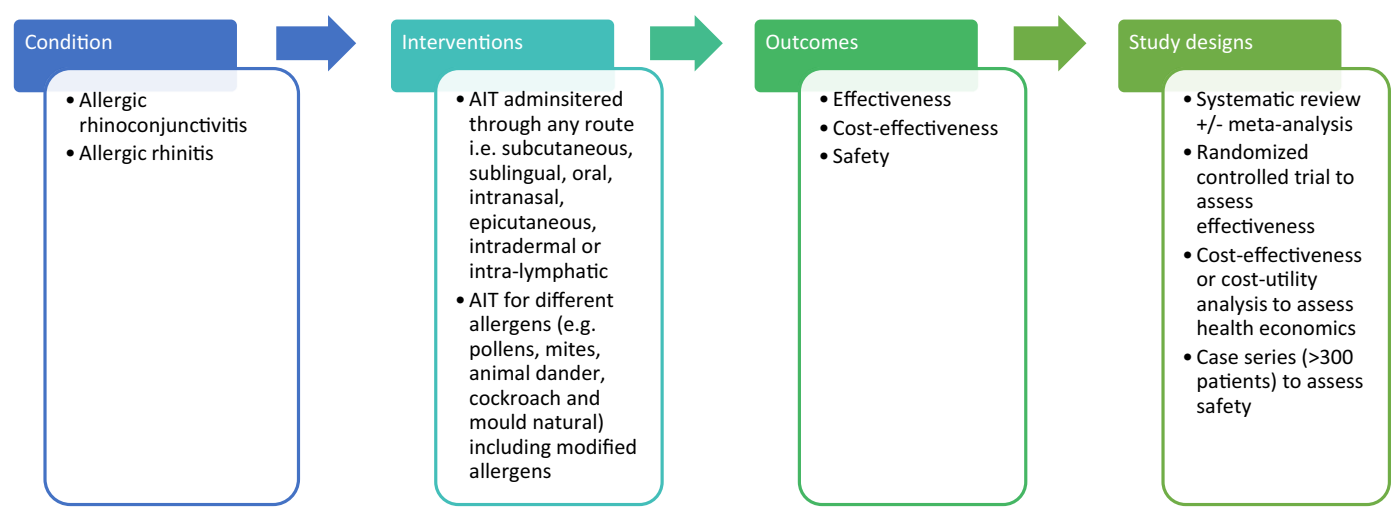

Fig. 1 Conceptualization of systematic review of allergen immunotherapy for allergic rhinoconjunctivitis 


\section{Inclusion criteria}

\section{Patient characteristics}

We will focus on studies conducted on patients of any age with a physician-confirmed diagnosis of allergic rhinoconjunctivitis or allergic rhinitis, plus evidence of clinically relevant allergic sensitization (e.g., skin prick test or specific-IgE).

\section{Interventions of interest and comparator}

This review is focused on AIT for different allergens (e.g. pollens, house dust mites, animal dander, cockroach and moulds), including modified allergens, administered through any route [e.g. subcutaneous (SCIT), sublingual (SLIT), oral, intranasal, epicutaneous, intradermal or intra-lymphatic] compared with placebo or any active comparator.

\section{Study designs}

Systematic reviews of RCTs and RCTs will be used to investigate effectiveness, health economic analysis will be used to assess cost-effectiveness, and systematic reviews, RCTs and case series with a minimum of 300 patients will be used to assess safety (smaller case series are excluded in order to minimise selection biases).

\section{Study outcomes}

Primary

- Effectiveness, both short-term (i.e. during treatment) and long-term (i.e. at least a year after discontinuation of AIT) assessed by symptom and/or medication scores [16].

\section{Secondary}

- Assessment of disease specific quality of life

- Threshold of allergen exposure to trigger symptoms in an environmental exposure chamber or allergen challenge

- Safety as assessed by local and systemic reactions in accordance with the World Allergy Organization's grading system of side-effects $[17,18]$

- Health economic analysis from the perspective of the health system/payer.

\section{Exclusion criteria}

The following exclusion criteria will be applied:

- Reviews, discussion papers, non-research letters and editorials

- Animal studies
- Quantitative studies not employing systematic review or RCT techniques

- Qualitative studies

- Case series (less than 300 patients).

\section{Study selection}

All references will be uploaded into the systematic review software Distiller and undergo initial deduplication. Study titles will be independently checked by two reviewers according to the above selection criteria and categorized as: included, not included or unsure. For those papers in the unsure category, we will retrieve the abstract and re-categorize as above. Any discrepancies will be resolved through discussion and, if necessary, a third reviewer will be consulted. Full text copies of potentially relevant studies will be obtained and their eligibility for inclusion independently assessed. Studies that do not fulfil all of the inclusion criteria will be excluded.

\section{Quality assessment strategy}

Quality assessments will independently be carried out on each study by two reviewers using the relevant version of the Critical Appraisal Skills Programme (CASP) quality assessment tool for systematic reviews and health economic evaluations [19, 20]. RCTs will be assessed for generation of allocation sequence, concealment of allocation, baseline outcome measurements, baseline characteristics, incomplete outcome data, blinding of outcome assessor, protection against contamination, selective outcome reporting and other risks of bias using the Cochrane Risk of Bias Tool. Similarly, we will use the quality assessment form produced by the National Institute for Health and Clinical Excellence (NICE) to critically appraise case series [21]. Any discrepancies will be resolved by discussion or, if agreement cannot be reached, a third reviewer will arbitrate.

\section{Data extraction, analysis and synthesis}

Data will be independently extracted onto a customized data extraction sheet in Distiller by two reviewers, and any discrepancies will be resolved by discussion or, if agreement cannot be reached, by arbitration by a third reviewer.

A descriptive summary with summary data tables will be produced to summarize the literature. If clinically and statistically appropriate, meta-analysis using either fixedeffect or random-effects modeling will be undertaken [16]. A narrative synthesis of the data will also be undertaken. It is expected that it will only be appropriate to include the SLIT and SCIT studies in a meta-analysis to minimize heterogeneity; studies using other approaches will be described narratively. 


\section{Sensitivity and subgroup analyses, and assessment for publication bias}

Sensitivity analyses will be undertaken by comparing the summary estimates obtained by excluding studies considered to be at high risk of bias with those considered to be at low or moderate risk of bias.

Subgroup analyses will be undertaken to compare:

- Children (5-11) versus adolescents (12-17) versus adults ( $\geq 18$ years)

- SCIT versus SLIT AIT

- Mild-to-moderate versus severe disease

- Aqueous solutions versus tablets in SLIT

- Modified allergen extracts (allergoids) versus unmodified allergen extracts in SCIT

- The use of single versus multiple allergens from different biological families in an extract

- AIT for seasonal versus perennial allergens

- Pre-seasonal (short term treatment) versus continuous treatment in SCIT

- Pre-/co-seasonal (short term treatment) versus continuous treatment in SLIT.

Where possible, publication bias will be assessed through the creation of funnel plots, and tested by Egger's regression test and Begg's rank correlation test [22, 23].

\section{Registration and reporting}

This review will be registered with the International Prospective Register of Systematic Reviews (PROSPERO): http://www.crd.york.ac.uk/prospero/. The Preferred Reporting Items for Systematic Reviews and Meta-Analyses (PRISMA) checklist will be used to guide the reporting of the systematic review: http://www.prisma-statement.org/.

\section{Discussion}

This review will involve systematically identifying, critiquing and synthesizing the evidence on the effectiveness, cost-effectiveness and safety of AIT for the management of allergic rhinoconjunctivitis. The review will take advantage of and build on other recent systematic reviews [24-27]. The findings from this review will be used to inform the development of recommendations for EAACI's Guidelines on AIT. We anticipate that this review will report in 2016.

\section{Authors' contributions}

This protocol was drafted by SD and the search strategy was developed by UN. It was initially revised following critical review by AS, GR and OP, and then by all co-authors. All authors read and approved the final manuscript.

\section{Author details}

${ }^{1}$ Evidence-Based Health Care Ltd, Edinburgh, UK. ${ }^{2}$ Systematic Review at Decision Resources Group Abacus International, Bicester, UK. ${ }^{3}$ The David Hide
Asthma and Allergy Research Centre, St Mary's Hospital, Newport Isle of Wight, NIHR Respiratory Biomedical Research Unit, University Hospital Southampton NHS Foundation Trust, University of Southampton, Southampton, UK. ${ }^{4}$ Faculty of Medicine, University of Southampton, Southampton, UK. ${ }^{5}$ Department of Otorhinolaryngology, Head and Neck Surgery University Hospital, Mannheim and Center for Rhinology and Allergology, Wiesbaden, Germany. ${ }^{6}$ Food Allergy Referral Centre Veneto Region, Department of Women and Child Health, Padua General University Hospital, Padua, Italy. ${ }^{7}$ Hospital Quiron Bizkair, Bilbao, Spain. ${ }^{8}$ National Heart and Lung Institute, Imperial College, London, UK. ${ }^{9}$ Department of ENT, Eskisehir Osmangazi University Medical Faculty, Eskisehir, Turkey. ${ }^{10}$ University and Hospital of Montpellier, Inserm Paris Sorbonnes, Montpellier, France. ${ }^{11}$ Section of Allergology, Department of Internal Medicine, Erasmus MC, Rotterdam, The Netherlands. ${ }^{12}$ Hans Christian Andersen Children's Hospital, Odense University Hospital, Odense, Denmark. ${ }^{13}$ Children's Center Bethel, EvKB, Bieledelf and Allergy Center Buhr-University, Bochum, Germany. ${ }^{14}$ Laboratory of Experimental Immunology, University Hospitals Leuven, Louvain, Belgium. ${ }^{15}$ ALC, Allergy Learning and Consulting, Copenhagen, Denmark. ${ }^{16}$ University Medical Center, Utrecht, The Netherlands. ${ }^{17}$ Hospital Medica Sur, Mexico City, Mexico. ${ }^{18}$ Department of Otolaryngology-Head and Neck Surgery, John Hopkins, Baltimore, USA. ${ }^{19}$ Allergy and Clinical Immunology Unit, 2nd Department of Pediatrics, University of Athens, P and A Kiriakou Children's Hospital, Athens, Greece. ${ }^{20}$ Department of Allergology, Groningen Research Institute for Asthma and COPD (GRIAC), University Medical Center Groningen, University of Groningen, Groningen, The Netherlands. ${ }^{21}$ Department of Pediatrics, University of Messina, Messina, Italy. ${ }^{22}$ Department of Pediatrics, Nippon Medical School, Tokyo, Japan. ${ }^{23}$ University of Milano, Milan, Italy. ${ }^{24}$ Department of Nutrition and Dietetics, Harokopio University, Athens, Greece. ${ }^{25}$ The Royal National Throat, Nose and Ear Hospital, University College London, London, UK. ${ }^{26}$ Netherlands Anafylaxis Network, Dordrecht, The Netherlands. ${ }^{27}$ Charite University Hospital, Berlin, Germany. ${ }^{28}$ Dept. of Paediatrics, Respiratory and Allergic Disease Division, Medical University Graz, Graz, Austria. ${ }^{29}$ Pharmaceutical Group of the European Union, Brussels, Belgium. ${ }^{30}$ Guy's and St Thomas' NHS Foundation Trust, London, UK.

${ }^{31}$ Chartie-Universitatsmedizin, Berlin, Germany. ${ }^{32}$ Beijing Institute of Otolarygology, Beijing, China. ${ }^{33}$ Allergy and Respiratory Research Group, The University of Edinburgh, Edinburgh, UK.

\section{Acknowledgements}

The EAACI Rhinoconjunctivitis AIT taskforce would like to thank Daniela Brombin for her administrative assistance and Stefan Kuzmiak for his assistance with information technology support for the activity.

\section{Competing interests}

S. Dhami: support to co-ordinate the undertaking of the systematic review; U. Nurmatov: support to undertake the review; G. Roberts: materials for research programme (ALK-Abello), research grant (ALK-Abello), advisory board (ALK-Abello), speaker (Allergy Therapeutics, ALK-Abelo); O. Pfaar: research grants, advisor and/or speaker (ALK Abelló, Allergopharma, Stallergenes, HAL Allergy, Artu Biologicals, Allergy Therapeutics/Bencard, Hartington, Lofarma, Novartis/Leti, GlaxoSmithKline, Essex Pharma, Cytos, Curalogic, Roxall, Biomay, Thermo Fisher, Circassia, European Union (FP-7 Health-2013 Innovation 1), Biotech Tools s.a., and Meda Pharma GmbH. Travel grants (HAL Allergy and Allergopharma). Advisor (Bencard, HAL Allergy, Novartis/Leti, Meda, ALK Abelló, Allergopharma, Biotech Tools s.a., GfK Bridgehead, Navigant Consulting, Sanofi, Guidepoint Global Advisors, Thermo Fisher and Stallergenes). Scientific Board Member of Mobile Chamber Experts (MCX), a GA2LEN Partner; A Muraro: Acting in consulting capacity for ALK, Meda Pharma, Nestle, Nutricia, Novartis. Grants from: Nestlé: Co-investigator for research protocol, Nutricia: Co-investigator for research protocols; IJ. Ansotegui: none; M. Calderon: lectures honorarium (ALK, Stallergens, Merck and Allergopharma), consultancy honorarium (ALK, Stallergenes and Hal); C. Cingi: none; P. Demoly: advisory board (ALK-Abello, Allergopharma, Cirassia, Chiesi, DBV, Stallergens, Thermo Fisher Scientific); S. Durham: advisory board (Merck), research grant or materials for research programme (ALK-Abelo, Merck, Regeneron, Biotools); R. Gerth van Wijk : research grant (ALK Abello, DBV); S. Halken: GAP study Steering (ALK-Abello); E.Hamelmann: research grant (AllergoPharma); P. Hellings: research grants (ALK-Abello, GCK) and committee membership (MEDA, Stallergens, GSK); L. Jacobsen: consulting (EAMG, ALK), travel support (EAMG), stock (>5000 euro)(ALK); E. Knol: Grant (HAL Allergy, Numico Research), consulting (HAL Allergy); D. Larenas-Linnemann: none; S. Lin: none; V.Maggina: none; H.Oude-Elberlink: financial support for Dutch Mastocytosis 
Centre (ALK-Abello); G. Pajno: research grant (Stallergens); R. Panwankar: none; E. Pastorello: none; C. Pitsios: none; G. Rotiroti: research grant (ALK-Abello); F.Timmermans: funding for Netherlands Anafylaxis Network (ALK-Abello, MEDA); O.Tsilochristou: none; EM. Varga: research grant (ALK-Abello), advisory committee (ALK-Abello); J. Wilkinson: none; A. Williams: none; M. Worm: advisory committee (ALK-Abello, Allergopharma, Bencard, Novartis, Meda); L. Zhang: none; A. Sheikh: support to co-ordinate the undertaking of the systematic review and development of the guidelines.

Funding

EAACl

\section{Appendix 1: Search strategy Search strategy 1 (MEDLINE, EMBASE)}

1. exp Rhinitis/

2. Rhinitis Allergic Perennial/

3. Rhinitis, allergic, seasonal/

4. hayfever.mp.

5. hay fever.mp.

6. fever, hay.mp.

7. seasonal allergic rhinitis.mp.

8. allergic rhinitides.mp.

9. allergic rhinitis.mp.

10. rhiniti".mp.

11. pollinosis.mp.

12. pollenosis.mp.

13. exp Nasal obstruction/

14. Conjunctivitis/

15. Conjunctivitis, Allergic/

16. conjunctivit".mp.

17. rhino-conjunctivit".mp.

18. or $/ 1-17$

19. exp Desensitization, Immunologic/

20. exp Immunotherapy/

21. Desensitization.mp.

22. Immunotherapy.mp.

23. Oral Immunotherapy.mp.

24. Oral desensitization.mp.

25. Sublingual Immunotherapy.mp.

26. Subcutaneous Immunotherapy.mp.

27. Epicutaneous Immunotherapy.mp.

28. Intradermal Immunotherapy.mp.

29. (Intra-lymphatic or intra lymphatic immunotherapy).mp.

30. Intranasal Immunotherapy.mp.

31. Specific Immunotherapy.mp.

32. Or/19-31

33. exp Intervention Studies/

34. Intervention Studies.mp.

35. Experimental stud".mp.

36. exp Clinical Trial/

37. Trial.mp.

38. Clinical Trial.mp.

39. exp Controlled Clinical Trial/

40. Controlled Clinical Trial.mp.
41. Randomi?ed Controlled Trial.mp.

42. exp Placebos/

43. Placebos.mp.

44. exp Random Allocation/

45. Random Allocation.mp.

46. exp Double-Blind Method/

47. Double-Blind Method.mp.

48. Double-Blind design.mp.

49. exp Single-Blind Method/

50. Single-Blind Method.mp.

51. Single-Blind design.mp.

52. Triple-Blind Method.mp.

53. Random*.mp.

54. Search:.tw

55. Review.pt.

56. Systematic review.tw.

57. Meta analysis.mp,pt.

58. Case series.mp.

59. (Case $\$$ and series).tw.

60. Cost:.mp.

61. Cost effective:.mp

62. Exp Health Care Costs/

63. (Costs and Costs Analysis).mp.

64. Economic evaluation".mp.

65. ((cost effective* adj1 analys*) or cost minimi?ation analys" or cost benefit analys" or cost utility analys* or cost consequence analys" or finances).mp.

66. Or/33-65

67. 18 and 32 and 66

\section{Search strategy 2}

(Cochrane library, TRIP, CINAHL, ISI Web of Science, HTA, EED) (Rhinitis* or allergic rhinitis or allergic rhinitides or seasonal allergic rhinitis or hayfever or hay fever or poll?nosis or pollenosis or conjunctivit* or allergic conjunctivitis or rhino conjunctivitis or rhino-conjunctivitis) and (Immunologic, desensiti* or immunotherapy or oral immunotherapy or oral desensiti?ation or sublingual immunotherapy or subcutaneous immunotherapy or epicutaneous immunotherapy or intradermal immunotherapy or intra-lymphatic immunotherapy or intranasal immunotherapy) and (Intervention stud" or experimental stud* or trial or clinical trial* or controlled clinical trial or randomi* controlled trial or random allocation or single blind method or double blind method or triple blind method or random* or systematic review or meta-analysis or case series or economic evaluation" or cost effective* analys" or cost minimi?ation analys* or cost benefit analys* or cost utility analys" or cost consequence analys* or finances)

Received: 25 November 2015 Accepted: 31 January 2016

Published online: 22 March 2016 


\section{References}

1. Mallol J, Crane J, von Mutius E, Odhiambo J, Keil U, Stewart A, ISAAC Phase Three Study Group. The International Study of Asthma and Allergies in Childhood (ISAAC) Phase Three: a global synthesis. Allergol Immunopathol. 2013;41:73-85.

2. Patil VK, Kurukulaaratchy RJ, Venter C, Grundy J, Roberts G, Dean T, et al. Changing prevalence of wheeze, rhinitis and allergic sensitisation in late childhood: findings from 2 Isle of Wight birth cohorts 12 years apart. Clin Exp Allergy. 2015;45:1430-8.

3. Bousquet PJ, Demoly P, Devillier P, Mesbah K, Bousquet J. Impact of allergic rhinitis symptoms on quality of life in primary care. Int Arch Allergy Immunol. 2013;160:393-400. doi:10.1159/000342991.

4. Brożek Jan L, Bousquet Jean, Baena-Cagnani Carlos E, Bonini Sergio, Canonica GW, Casale TB, van Wijk RG, Ohta K. Allergic Rhinitis and its Impact on Asthma (ARIA) guidelines: 2010 Revision. J Allergy Clin Immunol. 2010;126(3):p466-76.

5. Skoner DP. Allergic rhinitis: definition, epidemiology, pathophysiology, detection, and diagnosis. J Allergy Clin Immunol. 2001;108(1 Suppl):S2-8.

6. Walker S, Khan-Wasti S, Fletcher M, Cullinan P, Harris J, Sheikh A. Seasonal allergic rhinitis is associated with a detrimental effect on examination performance in United Kingdom teenagers: casecontrol study. J Allergy Clin Immunol. 2007;120(2):381-7.

7. Blanc PD, Trupin L, Eisner M, Earnest G, Katz PP, Israel L, et al. The work impact of asthma and rhinitis: findings from a population-based survey. J Clin Epidemiol. 2001;54:610-8.

8. Guerra S, Sherrill DL, Martinez FD, Barbee RA. Rhinitis as an independent risk factor for adult-onset asthma. J Allergy Clin Immunol. 2002;109(3):419-25.

9. Sheikh A, Singh Panesar S, Dhami S, Salvilla S. Seasonal allergic rhinitis in adolescents and adults. BMJ Clin Evid. 2007; pii: 0509.

10. Bousquet J, Khaltaev N, Cruz AA, Denburg J, Fokkens WJ, Togias A, et al. Allergic Rhinitis and its Impact on Asthma (ARIA) 2008 update (in collaboration with the World Health Organization, GA(2)LEN and AllerGen). Allergy. 2008;63(Suppl 86):8-160.

11. Zuberbier T, Bachert C, Bousquet PJ, Passalacqua G, Walter Canonica G, Merk $\mathrm{H}$, et al. GA ${ }^{2}$ LEN/EAACI pocket guide for allergen-specific immunotherapy for allergic rhinitis and asthma. Allergy. 2010;65:1525-30.

12. Walker SM, Durham SR, Till SJ, Roberts G, Corrigan CJ, Leech SC, et al. Immunotherapy for allergic rhinitis. Clin Exp Allergy. 2011;41:1177-200.

13. Pfaar $O$, Bachert C, Bufe A, Buhl R, Ebner C, Eng P, Friedrichs F, Fuchs $T$, Hamelmann E, Hartwig-Bade D, Hering T, Huttegger I, Jung K, Klimek L, Kopp MV, Merk H, Rabe U, Saloga J, Schmid-Grendelmeier P, Schuster A, Schwerk N, Sitter H, Umpfenbach U, Wedi B, Wöhrl S, Worm M, KleineTebbe J. Guideline on allergen-specifc immunotherapy in IgE mediated allergic diseases_- 22 k Guideline of the German Society for Aller gology and Clinical Immunology (DGAKI), the Society for Pediatric Allergy and Environmental Medicine (GPA), the Medical Association of German Allergologists (AeDA), the Austrian Society for Allergy and Immunology (ÖGAI), the Swiss Society for Allergy and Immunology (SGAI), the German Society of Dermatology (DDG), the German Society of Oto Rhino-Laryngology, Head and Neck Surgery (DGHNO-KHC), the German Society of Pediatrics and Adolescent Medicine (DGKJ), the Society for Pediatric Pneumology (GPP), the German Respiratory Society (DGP), the German Association of ENT Surgeons (BV-HNO), the Professional Federation of Paediatricians and Youth Doctors (BVKJ), the Federal Association of Pulmonologists (BDP) and the German Dermatologists Association (BVDD). Allergo J Int. 2014:23:282-319.

14. Hellings PW, Fokkens WJ, Akdis C, Bachert C, Cingi C, Dietz de Loos D, et al. Uncontrolled allergic rhinitis and chronic rhinosinusitis: where do we stand today? Allergy. 2013;68:1-7.

15. Pfaar O, Demoly P, van Gerth Wijk R, Bonini S, Bousquet J, Canonica GW, Durham SR, Jacobsen L, Malling HJ, Mösges R, Papadopoulos NG, Rak S, del Rodriguez Rio P, Valovirta E, Wahn U, Calderon MA. Recommendations for the standardization of clinical outcomes used in allergen immunotherapy trials for allergic rhinoconjunctivitis: an EAACI Position Paper. Allergy. 2014;69(7):854-67.

16. Higgins JPT, Green S (editors). Cochrane Handbook for Systematic Reviews of Interventions Version 5.1.0 [updated March 2011]. The Cochrane Collaboration, 2011. http://www.cochrane-handbook.org Last Accessed on 3rd Sep 2015.

17. Giovanni Passalacqua MD, Carlos E. Baena-Cagnani MD, Jean Bousquet MD, Giorgio Walter Canonica MD,Thomas B. Casale MD, Linda Cox MD, Stephen R. Durham MD, Desire Larenas-Linnemann MD, Dennis Ledford MD, Ruby Pawankar MD, Paul Potter MD, Nelson Rosario MD, Dana Wallace MD, Richard F. Lockey MD. Grading local side effects of sublingual immunotherapy for respiratory allergy: Speaking the same language http://www.jacionline.org/article/S0091-6749(13)00528-9/pdf.

18. World Allergy Organization Subcutaneous Immunotherapy Systemic Reaction Grading System https://www.aaaai.org/Aaaai/media/MediaLibrary/PDF\%20Documents/Immunotherapy\%20Forms/7b-World-AllergyOrganization-Systemic-Reaction-Grading-systemx.pdf.

19. CASP checklist for systematic reviews. http://media.wix.com/ugd/ dded87_a02ff2e3445f4952992d5a96ca562576.pdf. Last Accessed on 13th Nov 2015.

20. CASP checklist for Economic evaluations http://media.wix.com/ugd/dde d87_3b2bd5743feb4b1aaac6ebdd68771d3f.pdf Last accessed on $3^{\text {rd }}$ September 2015.

21. Higgins JPT, Green S. Cochrane Handbook for Systematic Reviews of Interventions. Version 5.0.2 (Chapter 11, Section 11).

22. Egger M, Davey Smith G, Schneider M, et al. Bias in meta-analysis detected by a simple, graphical test. BMJ. 1997;315:629-34.

23. Begg CB, Mazumdar M. Operating characteristics of a rank correlation test for publication bias. Biometrics. 1994;50:1088-101.

24. Calderon MA, Penagos M, Sheikh A, Canonica GW, Durham S. Sublingual immunotherapy for treating allergic conjunctivitis. Cochrane Database Syst Rev. 2011;7:CD007685.

25. Radulovic S, Calderon MA, Wilson D, Durham S. Sublingual immunotherapy for allergic rhinitis. Cochrane Database Syst Rev. 2010;12:CD002893.

26. Calderon MA, Alves B, Jacobson M, Hurwitz B, Sheikh A, Durham S. Allergen injection immunotherapy for seasonal allergic rhinitis. Cochrane Database Syst Rev. 2007;24(1):CD001936.

27. Nelson H, Cartier S, Allen-Ramey F, Lawton S, Calderon MA. Network meta-analysis shows commercialized subcutaneous and sublingual grass products have comparable efficacy. J Allergy Clin Immunol Pract. 2015;3(256-266):e3. 